\title{
Current perspectives on the safe electrical stimulation of peripheral nerves with platinum electrodes
}

\author{
Alexander R. Harris* \\ ARC Centre of Excellence for Electromaterials Science, Intelligent Polymer Research Institute, \\ University of Wollongong, NSW 2522, Australia.
}

Email: alexrharris@gmail.com

\begin{abstract}
This review details some PNS targets and electrode designs used for electrical stimulation. It investigates limitations in current knowledge of safe electrical stimulation and possible future electrode developments. Current PNS targets are large, leading to poor resolution and off-target side effects. Most clinical devices are platinum or platinum/iridium embedded in an insulation material. Their safety is usually guided by the Shannon plot, which is not valid for the PNS. New electrode designs are needed to target smaller nerve fibres, enabling higher resolution electrical therapies with fewer off-target side effects. Damage can occur through biological and electrochemical mechanisms. Greater mechanistic understanding is required to ensure safe and efficacious, long term electrical stimulation with new electrode materials, geometries and stimulation waveforms.
\end{abstract}

\section{Keywords}

Platinum; electrical stimulation; peripheral nervous system; safe stimulation protocol; electrochemical mechanisms

\section{Body of Article}

The peripheral nervous system (PNS) acts as a signal relay between the central nervous system (CNS) and the limbs and organs (Figure 1). The PNS is composed of somatic (voluntary) and autonomic (selfregulated) systems. Afferent or sensory nerves of the somatic system are excited by various stimuli, transferring information to the brain, while efferent nerves send signals from the CNS to muscles and organs. The autonomic system is often divided into 2 subsystems, the sympathetic and parasympathetic nervous systems, often simplified as quick and slow response systems respectively. Most of the PNS involves single nerve fibres; however the autonomic efferent pathway is composed of 2 sequential nerves, a preganglionic and postganglionic nerve that connects to the target organ. The cervical vagus nerve is a mixture of afferent and efferent, myelinated and unmyelinated nerve types which connect to several organs and muscles. The nerve fibre in adults is $\sim 3.78-4.79 \mathrm{~mm}$ in diameter [1], branching to increasingly smaller nerve fibres at their distal ends. The diameter of individual nerves varies, with motor neurons having the largest diameter and sensory nerves the smallest. The cervical vagus nerve is composed of 10-15 fascicles, however their structure varies between individuals and is still poorly understood [2].

The connection between the senses, organs and limbs to the CNS offers opportunities for interrogating and controlling their function. This short review will provide an overview of the current PNS targets being accessed for clinical applications and the electrode designs. It will then highlight some limitations in these approaches demanding higher resolution devices. These new devices will require greater understanding of the biological and electrochemical mechanisms involved in neural stimulation, ensuring safe and efficacious chronic performance. 


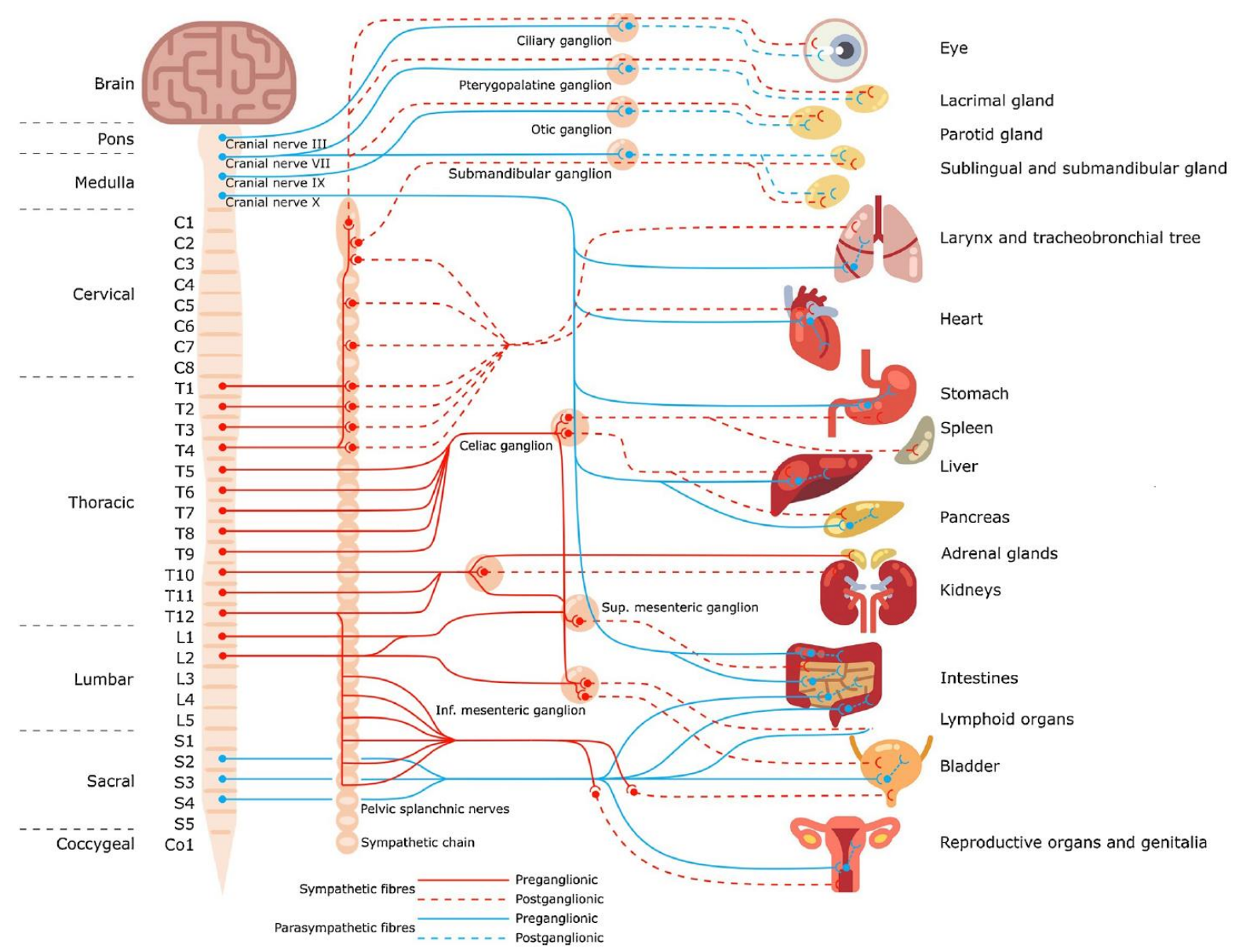

Figure 1: General scheme of the autonomic nervous system. Reprinted with permission from [2]

\section{Peripheral Nerve Targets for Electrical Stimulation}

Information travels along nerve fibres via a propagating action potential. The action potential is a rise of membrane potential from a resting potential between -40 and $-80 \mathrm{mV}$ to approximately $40 \mathrm{mV}$ and subsequent hyperpolarisation to around $-90 \mathrm{mV}$ before returning to the resting potential. The change in membrane potential is caused by the opening of ion channels and movement of ions across the cell membrane.

Initiation of an action potential is achieved naturally by many stimuli including chemical, optical, acoustic, mechanical and thermal mechanisms. It is also possible to induce an action potential by application of an electrical field from an external power source. The first demonstration of electrical stimulation of tissue is commonly recognised as Luigi Galvani in the 1780s when he used static electricity to induce movement in a dead frog leg. Subsequently, Alessandro Volta placed two metal rods into his ears and connected them to a battery. He sensed a shock within his head followed by the sound of boiling thick soup [3].

One of the initial uses of direct electrical stimulation of the PNS to provide therapeutic benefit was performed on the phrenic nerve in 1950 by Stanley Sarnoff [4]. The stimulation led to movement of the diaphragm, controlling ventilation. Diaphragm pacing is now used to treat high spinal cord injuries, paralysed diaphragm and central sleep apnoea.

Delivery of therapeutic sensory input was first performed in the cochlea. In 1957, Djourno and Eyriès implanted a single channel device into the temporal bone of a deaf patient and was able to provide auditory cues [5]. However the device failed after a few months due to breakage of the solder joint. The electrode may have also been stimulating the cochlear nucleus rather than the cochlear nerve fibres [6]. Beginning in 1961, William House developed the first FDA approved single channel cochlear 
implant that was implanted in over 1000 people [7]. Graeme Clark then led a team to create the first multi-channel device [8]. Cochlear implants have now been implanted in over 400,000 patients worldwide, providing significant improvements in speech perception to the profoundly deaf.

The first demonstration of functional electrical stimulation to improve body movement was performed by Liberson in 1961. He stimulated the peroneal nerve to improve the gait pattern of stroke patients [9]. Since then, a number of clinical devices have been developed for the treatment of movement disorders including spinal cord injury, stroke, foot drop and multiple sclerosis.

In 1967, electrical stimulation of the spinal cord was shown to inhibit pain for 1 and a half days before the patient deceased [10]. Many spinal cord stimulators are now available for the treatment of failed back surgery syndrome, chronic pain, intractable angina and other pain conditions.

The ability of electrical current to induce a phosphene was first reported by Charles Le Roy in 1755, by wrapping a wire around the head of a blind man [11]. Early efforts to create a vision prosthesis focussed on stimulating the visual cortex. More recently, devices have been created to stimulate the retina of blind patients, with the Argus II being the first to receive FDA approval [12].

Vagus nerve stimulation was first conducted by James Corning in 1884 for the treatment of epilepsy but with limited success [13]. It has now been reported to treat a range of conditions including depression, obesity and epilepsy [14]. Electroceuticals are a growing area of interest to selectively stimulate nerves within the body to control organ function [15]. The Electrical Prescriptions (ElectRx) program in Defense Advanced Research Projects Agency (DARPA) aims to demonstrate stimulation of the PNS can provide non-pharmacological treatments for medical conditions such as pain, rheumatoid arthritis, joint inflammation, post-traumatic stress disorder, irritable bowel disease and diabetes [16].

With over 200 years demonstrating electrical stimulation of the PNS can affect body function, it is now clear that it can provide sensory cues and treat intractable and drug resistant disorders (Table 1), however each of the above devices have limitations and inconsistent outcomes. For instance, patients receiving a cochlear implant generally have good speech perception, but music perception and appreciation is poor [17]. And stimulation of the entire vagus nerve results in significant off target side effects in organs not intended to be stimulated. Vagus nerve stimulation to control epileptic seizures often leads to hoarseness, throat pain, coughing, shortness of breath, tingling and muscle pain [18]. Eliminating these side effects is difficult as the mechanism of vagus nerve stimulation is unknown, with synchronisation/desychronisation of nerve activity, changes in blood flow and release of neurotransmitters all being proposed. It is believed that poor control of current from the electrodes to target neural tissue is a significant cause of these limitations and side effects. This includes variable neural structure and function between people, and different placement of electrodes. Therefore there is a need to target smaller, more distal and more homogeneous nerve fibres that are closer to the target organs to achieve more selective neural stimulation.

Table 1: Common Peripheral Nerve Targets for Electrical Stimulation and Associated Disease States

\begin{tabular}{|ll|}
\hline Nerve Target & Associated Disease States \\
\hline Auditory Nerve & Profound hearing loss \\
\hline Retinal Nerves & Partial or total blindness, retinitis pigmentosa, macular degeneration \\
\hline Phrenic Nerve & Diaphragm pacing, respiratory failure, sleep apnoea, spinal cord injury \\
\hline Hypoglossal Nerve & Sleep apnoea \\
\hline Occipital Nerve & Chronic migraine \\
\hline Sacral Nerve & Overactive bladder, incontinence, constipation, chronic pelvic pain \\
\hline Pudendal Nerve & Incontinence, urogenic, iliac crest and abdominal pain \\
\hline
\end{tabular}




\begin{tabular}{|ll|}
\hline Tibial Nerve & Overactive bladder, incontinence \\
\hline Peroneal Nerve & Foot drop \\
\hline Spinal Cord & $\begin{array}{l}\text { Chronic pain, failed back surgery syndrome. intractable angina, visceral } \\
\text { abdominal and perineal pain, pain in the extremities from nerve damage }\end{array}$ \\
\hline Trigeminal Nerve & Craniofacial pain \\
\hline Vagus Nerve & Intractable epilepsy, treatment resistant depression \\
\hline
\end{tabular}

\section{Electrode Designs for Peripheral Nerve Stimulation}

While electrical stimulation of the PNS aims to induce neural activity, the anatomy, tissue composition, nerve fibre type and neurite orientation vary significantly for each target. In general, the electrode should be placed as close to the target nerve as possible to ensure selective neural stimulation and minimise the required stimulation amplitude needed to pass through the resistive tissue. The stimulation threshold $\left(I_{\text {th }}\right)$ is a function of electrode-neuron distance $(r)$ with a current offset $\left(I_{\mathrm{R}}\right)$ and slope $(k)$ (equation 1) [19]. Therefore, clinical devices must be designed specifically for the anatomy of each tissue target.

$$
I_{t h}=I_{R}+k r^{2}
$$

Implanted devices must be biocompatible (not inducing an adverse response from the host) and biostable (maintain function over the device lifetime). Numerous reviews are available on the immune response to implants [20]. And significant effort has focussed on developing appropriate materials and device designs to ensure implanted electrodes are biocompatible and biostable [21]. Further research efforts will continue to improve the biocompatibility and biostability of implantable devices.

Electrodes providing sensory input are tailored to the anatomy of the sensing organ, with the aim of providing realistic percepts. The cochlear implant is typically composed of 12-24 platinum thin film electrodes embedded in silicone rubber [22][23]. The specific electrode size and geometry varies between devices and manufacturers but is typically a band or half band in the order of $0.3 \mathrm{~mm}^{2}$. The Argus II has 60, $200 \mu \mathrm{m}$ diameter platinum grey electrodes in a silicone substrate [24][25]. The Alpha IMS vision prosthesis is composed of $50 \times 50 \mu \mathrm{m}$ titanium nitride electrodes on a polyimide foil [26].

Spinal cord stimulators can be catheter like or paddles with a range of electrode sizes and number [27]. They are typically $3 \mathrm{~mm}$ long platinum/iridium foil electrodes encapsulated in silicone or polyurethane.

Vagus nerve stimulators and functional electrical stimulators may be implantable or transcutaneous. Transcutaneous electrodes are less invasive, reducing surgical risk, however the large electrode-neuron distance increases the power usage and chance of off target stimulation. Implantable electrodes are available in different geometries but are typically platinum in a silicone rubber insulation [28]. Cuff or coil electrode geometries wrap around a nerve fibre; more selective neural electrodes penetrate nerve fascicles, with longitudinal intrafascicular electrodes lying across a nerve fibre and transverse intrafascicular multichannel electrodes penetrating a nerve fibre [29]. However these penetrating electrodes are more invasive, inducing greater tissue damage and have poorer lifetimes. See [28] for a comprehensive review of electrode designs.

In general, most clinical implantable electrodes are platinum or platinum/iridium of varying size and geometry. The topography is usually relatively smooth, with platinum grey used on the Argus II having a higher surface roughness. Higher surface area platinum black has been tested in animal models but the initial electrochemical benefits appeared to decrease over time and so it wasn't translated to clinical devices [30]. Platinum particles have also been embedded in polydimethylsiloxane (PDMS) where they were used as a spinal cord stimulator in a mini pig model, but again the performance degraded over time [31].

Recently a new microelectrode was shown to have very high selectivity [32]. A carbon nanotube fibre coated with parylene was implanted into the tibial nerve of a rat (Figure 2). Electrical stimulation via 
needle electrodes in each toe induced compound action potentials, which were detected on the carbon nanotube fibre. The electrode had an increased selectivity index to adjacent toe stimulation compared to previously reported penetrating electrodes. A highly flexible, conductive graphene fibre capable of being tied into a tight knot has also been used to record activity from the cortex of a rat [33] and shows very high selectivity and signal-to-noise ratio from peripheral nerve recordings as a neural cuff electrode (Prof Mario Romero-Ortega; private correspondence).

The selectivity of neural stimulation can also be improved through tailoring of the stimulation waveform. Large fibre diameters $(D)$ have a lower threshold than smaller fibres (equation 2) [19], and short pulse biphasic waveforms with no interphase gap between cathodic and anodic pulses can enhance this effect [34]. This can allow stimulation of certain nerve types, such as motor or sensory neurons. Current steering through careful electrode and waveform design can therefore be used to preferentially stimulate certain nerve fibres.

$$
I_{t h}=I_{D}+\frac{a}{\sqrt{D}}
$$

Presently, platinum is used for most implantable charge delivery electrodes, as it is corrosion resistant, has a high conductivity and reasonable charge injection capacity. Its shortcomings include being a very expensive precious metal; it is hard and stiff, so that insertion and movement within soft, flexible tissue can result in loss of contact with the target cells, tissue damage and scarring; it has limited surface chemistry, preventing tailoring of cellular response with surface charge, topography and functionality; and the electrode impedance and charge injection capacity could be improved. This has led to significant efforts to develop new soft, functional, conductive materials to improve the electrode-tissue interface [35]. However, most of this research has focussed on the CNS rather than in the PNS. And most of these new materials have shown limited stability in vivo and after chronic electrical stimulation. As a result, platinum's long history in functional devices and as an FDA approved material still makes it the first choice when developing new clinical devices. Recent results with small, flexible, carbon based electrodes outperformed the selectivity and sensitivity of current clinical devices, opening up the possibility of a new generation of PNS interfacing devices. Development of new implantable devices raises questions about their safe performance during long-term stimulation. 


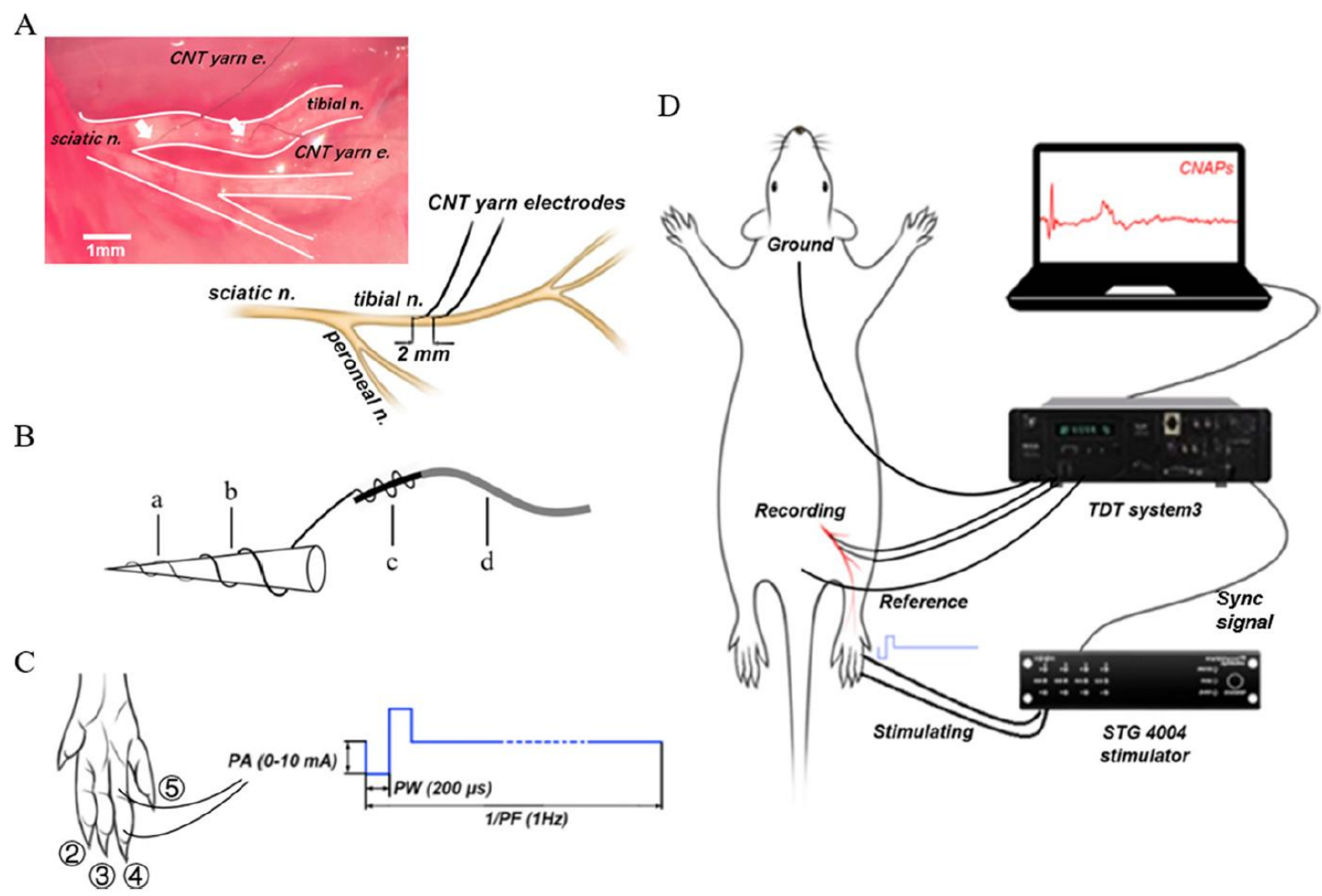

Figure 2: Acute peripheral nerve recordings. (A) Illustration of two CNT yarn electrodes separated by approximately $2 \mathrm{~mm}$ in the tibial nerve. The left insert of (A) shows a photograph of the implanted electrodes during the experiment. Two white arrows refer to the implantation sites of two CNT yarn electrodes with $\sim 500 \mu \mathrm{m}$ exposed length. (B) Schematic view of the CNT yarn electrode. The length of the needle tip is $1 \mathrm{~mm}$. a the uninsulated segment of the CNT yarn $(\sim 500 \mu \mathrm{m}), \mathrm{b}$ the insulated segment of the CNT yarn, $c$ the connection between the uninsulated segment of the CNT yarn and the stainless steel, which is insulated by Parylene-C, and $\mathrm{d}$ the Teflon-insulated stainless steel. (C) Schematic view of the hind paw of the rat. Electrical stimuli, with biphasic rectangular cathodal-first pulses of a fixed duration of $200 \mu$ s and frequency at $1 \mathrm{~Hz}$ in the range from 0.5 to $10 \mathrm{~mA}$ were delivered through needles in toes 2 to 5. (D) Experimental set-up. Reprinted with permission from [32]

\section{Safe Electrical Stimulation}

Implantation of an electrical stimulator can adversely affect the patient. Early reports on the histopathology of clinical PNS stimulators revealed several patients had suffered surgical trauma, infection, inflammation and mechanically induced tissue damage [36]. Development of better surgical techniques and device designs has significantly reduced these damaging mechanisms.

Charge delivery across the electrode-tissue interface must be performed at safe stimulation levels to prevent damage to the electrode or surrounding tissue. The safe charge injection limits of implantable electrodes is often guided by the Shannon plot [37]. The Shannon plot relates the charge density per phase $\left(\mu \mathrm{C} \mathrm{cm}{ }^{-2} \mathrm{ph}^{-1}\right)(D)$ and the charge per phase $\left(\mu \mathrm{C}\right.$ phase $\left.^{-1}\right)(q)$ of a biphasic current pulse. Shannon defined the equation

$$
\log D=k-\log (q)
$$

with $k<1.5$ displaying no tissue damage. The Shannon plot mainly used data from stimulation of disc shaped platinum electrodes placed on the surface of a feline cortex. The stimulation protocol was for 7 hours with $400 \mu$ s anodic first, symmetrical, charge balanced, biphasic pulses with no interphase gap and at 50 pulses per second [38][39]. A more recent analysis has shown the Shannon plot is not valid for many conditions outside this stimulation protocol, in particular different stimulation waveforms and on microelectrodes [40]. The tissue composition is different in the CNS than in the PNS, and the neural 
structure and density are much higher and more complex. McCreery et al noted that neural injury in the PNS was seen at lower charge densities than in the CNS using similar stimulation waveforms [41]. Therefore, the Shannon plot is not valid in the PNS and the safe stimulation protocols for different tissue, electrode materials and geometries and over long-term stimulation are not known. More fundamentally, the empirical observations of tissue damage observed in these studies was most likely due to biological mechanisms, however the safe stimulation limits associated with electrochemical and biological mechanisms haven't been isolated. Furthermore, the short-term histological studies don't take into account the effect of fibrotic tissue growth that occurs on most devices or detect electrode damage that can accumulate over years of electrical stimulation.

Safety studies of electrical stimulation in PNS by McCreery et al were performed with a cuff electrode on a cat peroneal or sciatic nerve. Stimulation for 8-16 hours at 50-100 Hz with charge balanced, biphasic $100 \mu \mathrm{s}$ pulses of 2,500 pA resulted in irreversible neural damage with endoneural oedema visible 48 hours after stimulation and early axonal degeneration (EAD, where the myelin collapsed into the axoplasmic space) occurring within a week [36]. The damage was uniform across the nerve fibre, mainly affecting the medium to large fibres. Damage was reduced by lowering the stimulation period, pulse frequency, pulse length and duty cycle [42]. The strongest correlation to EAD was seen with the stimulus current as a multiple of full axonal recruitment [41]. Due to the short time period used for the stimulation and concerns over the validity of the animal model to humans, a large safety margin in clinical stimulation parameters was recommended.

The safety of cochlear implants was also assessed in cats over several months [43]. The number of hair cells and spiral ganglion neurons was reduced by the implantation, but there was no change in electrically evoked auditory brainstem response (ABR). Only after hundreds of hours of electrical stimulation was the ABR reduced, and this was associated with bone growth and loss of nerve fibres near the electrodes. The electrically stimulated damage was detected after 200 hours of stimulation at $200 \mu \mathrm{C} \mathrm{cm}{ }^{-2} \mathrm{ph}^{-1}, 100 \mu \mathrm{s} \mathrm{ph}^{-1}$ charge balanced biphasic pulses at 20-25 pulses per second. Use of a charge imbalanced waveform significantly reduced the safe stimulation levels. Further studies demonstrated stimulation for 16 hours a day for 4 months using $26 \mu \mathrm{C} \mathrm{cm}^{-2} \mathrm{ph}^{-1}, 100 \mu \mathrm{s} \mathrm{ph}^{-1}$ charge balanced biphasic, bipolar pulses at 100 pulses per second (typical levels used in clinical devices and well below the Shannon limit) had no statistically significant effect on spiral ganglion fibre survival compared to non-stimulated cochleae [44]. This raised the possibility that therapeutic stimulation levels for cochlear implants are safe.

More recently, the temporal bone from the first multi-channel cochlear implant recipient was analysed after they had deceased [45]. The initial implantation was performed in 1978; it was replaced in 1983 and again in 1998 before they passed away in 2007. The analysis indicated platinum had detached from the electrode and particulates had built up in fibrous tissue around the implant. There was an estimated loss of $85-90 \%$ in spiral ganglion cells, but it is unknown if the fibrotic tissue growth, electrical stimulation or platinum corrosion played any part in the cell loss. In addition, it isn't known at what stage the particulates formed and if further corrosion or cell loss would occur over longer time periods. So while the stimulation protocol was considered safe, it is unclear if the electrical stimulation was either too conservative or unsafe over long-term use.

Application of stimulation levels above the Shannon limit was deliberately used on cochlear implants in guinea pigs over 4 weeks to determine its effects on surrounding tissue [46]. No surgical trauma was induced, indicating any subsequent tissue damage was caused by electrical stimulation. Fibrous tissue growth occurred on all implanted electrodes, but increased with applied charge density. Pitting of the platinum electrode was seen above the Shannon limit and platinum particles detected in the fibrous tissue next to the electrode. There was also a focal region of necrosis in the fibrous tissue at high charge densities. However high charge densities increased the number of nearby auditory neurons, which suggests electrical stimulation is a trophic factor and the $\sim 0.2-1.0 \mathrm{~mm}$ separation between the electrode and target neurons prevent stimulation induced neural damage.

Previous studies on long term safety of electrical stimulation of PNS was recently reviewed [47]. It was noted that stimulation parameters depend on the specific nerve types and their size. In a fibre containing multiple nerve types, therapeutic benefit may be achieved by stimulating one nerve type but 
be damaging to other adjacent nerves. In general, short pulse durations and low pulse frequencies are safer, but the safe pulse frequency in the PNS is lower than in the CNS. A short interpulse delay and small electrode-neuron distance reduces stimulation threshold, which lowers the necessary stimulation amplitude and potential for damage. However there is insufficient data in the literature for more specific safety guidelines. And due to the different nerve size and anatomy between species, great caution must be taken when applying animal studies to humans.

A new generation of small, flexible electrodes may reduce the foreign body response, maintaining small electrode-neuron distances during chronic implantation, resulting in sustained, lower stimulation thresholds. Electrodes targeting smaller nerve fibres will also have a smaller electrode-neuron distance, resulting in a more uniform current distribution across the fibre. This raises the possibility that these types of electrodes can provide safer stimulation with fewer off target side effects.

The safe electrical stimulation levels of implantable devices is guided by the Shannon plot, but it is not applicable to the PNS; there are no standard protocols for safety testing or clinical use of peripheral nerve stimulators [40]. Developing a new device for stimulating the PNS, such as carbon based fibres, and determining their safe stimulation levels requires significant pre-clinical testing. Any data obtained over a relatively short time frame and from various tissue models may be too conservative, a poor predictor of long-term safety or of limited relevance to other device designs and stimulation protocols. Therefore, a more theoretical basis for safe stimulation parameters would significantly reduce costs associated with clinical trials, reduce patient risk and potentially improve clinical outcomes. The first step in this process is to separate out the biological and electrochemical mechanisms of stimulation induced electrode and tissue damage.

\section{Biological Mechanisms of Unsafe Electrical Stimulation}

Implantation of an electrode leads to surgical trauma, ongoing inflammation and a fibrotic tissue response to the presence and movement of the large, stiff device [48]. The foreign body response can isolate the electrode from the target tissue, reducing device performance and subsequently requiring a larger stimulation charge to achieve therapeutic benefit. The trauma and fibrotic tissue response can also damage or kill the target neural tissue. To overcome these issues, there has been significant effort to assist in avoiding blood vessels during implantation, minimise device size, reduce its stiffness and incorporate chemical cues such as anti-inflammatories and neural growth factors. An ideal electrode would be invisible to the body and remain in close contact with the target neurons over its entire lifetime. Separation from this ideal situation limits our ability to understand the different mechanisms involved in the safe electrical stimulation of tissue.

It is assumed that electrical stimulation of a nerve alters the ion concentration across the cell membrane, rather than specifically activating cell receptors or inducing more complex mechanisms. The change in ion concentration raising the membrane potential to a threshold potential, inducing an action potential.

Cell death from electrical stimulation can occur through different mechanisms. If very high potentials are applied, electroporation can occur, where the lipid membrane morphology is rearranged, creating pores that disturb intracellular homeostasis. The pores may then heal or expand, leading to cell rupture. However the potentials required for electroporation are well above those used in current clinical devices and is unlikely to be a major factor.

Excitotoxity of neurons occurs through the over stimulation of cell receptors, leading to cell stress and death [49]. Application of a local anaesthetic during acute electrical stimulation of PNS prevented cell death, indicating this mechanism may occur [36]. The uniform damage across the nerve fibre, and greater impact on the more excitable medium to large fibres further supports an overstimulation mechanism was occurring with this electrode and stimulation waveform. Reducing the stimulation frequency can limit this damage mechanism, but may adversely affect neural selectivity. However, the specific biochemical mechanisms occurring during electrical stimulation of neurons has not been thoroughly investigated. So the wide applicability of excitotoxic mechanisms to different clinical devices during chronic use is unknown as are effects of variations in tissue type and stimulation waveform. 
When developing new electrodes for more selective stimulation of the PNS, biological mechanisms of tissue damage may occur. To understand the biological mechanisms occurring during electrical stimulation and potential damaging mechanisms, a range of new tissue models are required to assess the effect of electrical stimulation on cell behaviour. Due to differences in nerve fibre size, anatomy, genotype and disease pathogenesis, animal models have limited relevance to clinical studies in humans. And assessment of biological damage caused by clinical devices can usually only be performed postmortem. New in vitro models may provide more relevant testing methods. Human induced pluripotent stem cells (iPSCs) can be used to generate different tissue types and structures that are more clinically relevant. Electrodes placed into iPSC derived tissue models would enable the effect of different electrical stimulation protocols, electrode designs, materials, neural structure and electrode-neuron arrangement to be assessed systematically without the added complexity of a foreign body response. The use of human derived tissue models would reduce the number of poorly relevant animals used in preclinical research. These tissue models can also be personalised for individual patients, which may allow tailoring of devices or stimulation protocols for enhanced clinical outcomes. It may also indicate differences in biochemical pathways between clinical groups, for instance patients with specific neurodegenerative diseases may have altered cellular composition or lost certain nerve types resulting in different stimulation or damage thresholds and mechanisms.

\section{Electrochemical Mechanisms of Unsafe Electrical Stimulation}

Electrical stimulation of tissue requires charge to pass through the electrode-tissue interface. This charge can be capacitance (movement of ions), Faradaic (oxidation or reduction of electrochemically active species) or pseudocapacitance (oxidation or reduction of the surface layer of the electrode). Assuming the electrode is inert and charge delivery is purely capacitive, the biochemical mechanisms would be unaffected by electrode material. However no electrode is ideal, particularly in the complex tissue environment, so charge is passed through a mixture of capacitance and Faradaic reactions. Electrochemical reactions occurring at the electrode/tissue interface may generate cytotoxic species and the electrode may corrode.

Platinum can pass charge into tissue through capacitance and a range of Faradaic reactions including platinum oxide formation and removal, hydride adsorption and stripping, water electrolysis and platinum stripping [50]. Electrochemically induced damage at the electrode/tissue interface could include platinum corrosion and formation of cytotoxic species including platinum nanoparticles, platinum salts, hydrogen and oxygen gas, reactive oxygen species, changes in $\mathrm{pH}$ and reaction products from other biomolecules present including amino acids, proteins, neurotransmitters, sugars, phospholipids, DNA and RNA. There are numerous articles detailing various reactions occurring at a platinum-solution interface. The charge transfer mechanisms that occur, and their kinetics depend on the conditions at the electrode-tissue interface; electrode size, surface topography and crystal structure, solution composition and applied waveform [51][52].

Several studies have been performed to determine if electrochemical reactions at a platinum electrode can affect cell viability. Biphasic electrical stimulation of a cochlear implant in $0.5 \% \mathrm{NaCl}$ was performed for 600 hours at a duty cycle of $50 \%$, frequency of $5 \mathrm{kHz}, 100 \mu$ s pulse width with no interpulse delay and an amplitude of $0.3 \mathrm{~A}$ [53]. Strong corrosion of the electrode was found. Titrations of the platinum containing media were then fed to NIH3T3 and SH-SY5Y cell lines. Evidence of cytotoxic effects on both cell lines were seen at platinum concentrations significantly below cells cultured with platinum nanoparticles with an average particle size of $3 \mathrm{~nm}$.

Electrical stimulation was performed in cell media with a deep brain stimulation electrode using a charge balanced, cathodic first biphasic waveform, 50 pulses per second, $100 \mu$ s pulse width, with a $100 \mu$ s interpulse gap for 2 hours [54]. The stimulation amplitude was either below or above the Shannon limit at $4.7 \mathrm{~mA}(\mathrm{k}=0.57)$ or $35 \mathrm{~mA}(\mathrm{k}=2.3)$. Higher platinum dissolution occurred with increased stimulation amplitude. Feeding the platinum containing solution to BV-2 microglia displayed signs of cytotoxicity with increasing platinum concentration and exposure time. However NSC-34 neural cells fed the same media only showed a loss of neurites. Efforts to prevent platinum dissolution were then attempted by using charge imbalanced biphasic pulses [55]. By lowering the charge during the anodic pulse, the electrode polarisation can be kept below a certain potential. It was argued that 
tissue damage can only occur by platinum oxidation, and by lowering the anodic polarisation, cathodic charge densities above the Shannon limit can be used safely. However these experiments were performed for extremely short time periods of $20 \mathrm{~s}$, and no tissue studies were performed to demonstrate these stimulation waveforms are safe.

These studies indicate reaction products formed at a platinum electrode during electrical stimulation can be cytotoxic, but specific chemical species are to blame, and these details aren't known. The effect of different electrical stimulation conditions (changes to media, electrode design or stimulation waveform) on the types of chemical species produced hasn't been assessed. Furthermore, the impact of these chemical species on primary neural cells hasn't been tested.

Understanding the charge transfer mechanisms that occur during electrical stimulation of tissue can only be achieved with appropriate models. Many electrochemical studies are performed in very simple conditions which may not be relevant to clinical use. Early studies on the electrochemistry of platinum in phosphate buffered saline indicated charge balanced, anodic first, $400 \mu \mathrm{C} \mathrm{cm}^{-2}, 1 \mathrm{~ms}$ pulses at $50 \mathrm{~Hz}$ resulted in platinum dissolution [56]. Addition of human serum albumin significantly reduced the platinum dissolution while removal of oxygen and using a cathodic first pulse had a smaller reduction effect. These experiments were mostly performed on freshly platinised electrodes which were quite rough and probably had little oxide present [57]. Later electrochemical studies used cyclic voltammetry on acid cleaned electrodes and indicated various amino acids also adsorbed onto the electrode surface but that cysteine increased platinum dissolution [58]. While these studies indicate platinum corrosion can occur, the use of acid cleaned electrodes without a layer of oxide may affect the platinum dissolution rate and is a poor model of clinical performance. The ability of amino acids and proteins to adsorb onto the electrode was also found to affect the amount of platinum corrosion, but the type and concentration of organic species is not equivalent to those in tissue, further limiting the relevance of these studies. Studies have also shown platinum is capable of corroding through both oxidation and reduction based mechanisms, but these are often performed in high concentration $\mathrm{H}_{2} \mathrm{SO}_{4}$ solutions, and it is unknown if these mechanisms can occur with platinum in tissue [59]. A greater understanding of the variation in tissue composition and its effect on charge transfer reactions at the platinum-tissue interface is needed.

The use of inappropriate electrochemical methods can also provide incorrect charge transfer mechanisms and safe charge injection limits of functioning clinical devices [51]. Reactions that are thermodynamically feasible may have very slow kinetics or be irreversible [60]; and reactions that seem insignificant on a single cyclic voltammetric sweep may accumulate over multiple current pulses. For instance, a stimulation waveform may be a charge balanced biphasic pulse, but the reaction mechanisms over reduction and oxidation pulses may not be balanced. Multiple pulses can then drive large changes in the electrode/tissue interface, such as the removal of platinum oxide, and is termed ratcheting [52][61]. Simply measuring the charge injection capacity from the reduction sweep of a voltammogram or the change in electrode potential during current pulsing can be a very poor predictor of safe charge injection limits and long term stability.

The safe charge injection limits are typically measured between the water oxidation and reduction potentials assuming these reactions cause the most damage. The charge storage capacity is obtained from integrating a potential sweep during cyclic voltammetry, and the charge injection limit measured from the change in potential during a current pulse. However, if unsafe electrochemical mechanisms, such as platinum corrosion or formation of cytotoxic species, occur at smaller potentials than water oxidation or reduction, then the safe charge injection limit will be smaller than currently accepted. Changes to a platinum electrode geometry, topography, stimulation waveform and the composition of the tissue and bathing solution surrounding the electrode can affect the reaction mechanisms (eg rate of platinum dissolution or oxygen reduction) that occur during electrical stimulation. These reactions can also vary over time with changes to electrode structure or tissue composition. As a result, the electrochemical mechanisms that can occur on each clinical devices may be different. This will then affect their safe stimulation protocol. Further fundamental studies of the electrochemical behaviour of clinical devices in different tissues over time will determine what role electrochemically induced damage plays. 
Platinum corrosion can produce cytotoxic species, resulting in unsafe electrochemical mechanisms during neural stimulation. Changes to electrode materials, such as the use of carbon based electrodes may remove platinum from the device, eliminating concerns on electrode corrosion and platinum toxicity. This could result in a larger safe charge injection limit. A greater understanding of what reaction mechanisms occur at the electrode-tissue interface is needed to determine those that are cytotoxic and damage the electrode. This will then guide the next generation of electrode materials and stimulation waveforms for safe, long term electrical stimulation of the PNS.

\section{Conclusions}

Electrical stimulation of peripheral nerves is being used to treat a range of disorders. The majority of the electrodes used in clinical devices are platinum or platinum/iridium embedded in an insulating material. The growth in electroceuticals means new electrode designs are being developed for clinical use. The next generation of implantable electrodes will target smaller nerve fibres to reduce off target side effects. Developing new devices requires a large investment in pre-clinical empirical studies that have limited utility to long term safety in humans. The safe stimulation protocols used for these devices are guided by the Shannon plot, however it's applicability to different tissues, electrode designs and stimulation protocols over long term use is poor. A more fundamental understanding of electrical stimulation is required. Tissue damage can occur through biological and electrochemical mechanisms, but further work is required to separate out different mechanisms and how they change with tissue type and structure and over time. Understanding the effects of electrical stimulation on tissue and damage causing mechanisms requires more relevant testing models such as iPSC derived tissue.

\section{Future Perspectives}

A new generation of bionic devices targeting the PNS will be developed to treat intractable and drug resistant disorders and reduce off target side effects caused by current implantable devices. There are a growing range of electrode geometries, topographies and materials being investigated. It is expected that these devices will be tailored to specific tissues and will include much smaller electrode sizes, and softer, more flexible materials. There will also be a development of new fabrication methods and tailoring of devices through advanced fabrication techniques such as 3D printing. These devices will also include chemical functionality and drug release to reduce immune response to and enhance neural integration with the implanted device.

To ensure these devices are used in a safe manner, a greater understanding of charge transfer mechanisms occurring over time in different tissue, and with different electrode geometry, topography and materials will be undertaken. Use of iPSCs will enable creation of different tissue models for assessing electrical stimulation safety and efficacy. The biological and electrochemical mechanisms occurring during electrical stimulation will be investigated in more detail, providing a more fundamental understanding of electrical stimulation of neurons.

\section{Executive Summary}

\section{Peripheral Nerve Targets for Electrical Stimulation}

- Electrical stimulation of the PNS has a long history

- Clinical devices for electrical stimulation of the PNS were initially developed around 1950 and has been growing rapidly for novel treatments of intractable disorders

- A new generation of clinical devices are required to target different tissue and smaller nerve fibres

\section{Electrode Designs for Peripheral Nerve Stimulation}

- Electrodes used to stimulate the PNS are mostly platinum or platinum/iridium embedded in an insulating material

\section{Safe Electrical Stimulation}

- Early safety studies on PNS electrodes focussed on surgical trauma, infection, inflammation and mechanically induced tissue damage 
- The safe electrical stimulation of neurons is guided by the Shannon plot which is mainly based on empirical evidence of stimulating the CNS over a short time frame

- The Shannon plot is not valid for the PNS, different stimulation waveforms and on microelectrodes and does not take into account different tissue, electrode materials, geometries and long-term stimulation

- Induced damage can occur through biological and electrochemical mechanisms

- Induced damage may not be visible over short term testing and the mechanisms may change over time

\section{Biological Mechanisms of Unsafe Electrical Stimulation}

- Biological damage can occur through excitotoxic mechanisms, but it is unclear how applicable this mechanism is across different tissues and devices

- Better tissue models, such as iPSC derived neural constructs are needed to understand the safety and efficacy of electrical stimulation

\section{Electrochemical Mechanisms of Unsafe Electrical Stimulation}

- Electrochemical damage can occur through corrosion of platinum electrodes and possibly by formation of cytotoxic species.

- Specific chemical species are responsible for cytotoxicity.

- A greater understanding is required of the biological and electrochemical mechanisms occurring during electrical stimulation, this will better define the safe stimulation protocols used on different devices and in different tissue

\section{Acknowledgements}

Funding from the Australian Research Council Centre of Excellence Scheme (Project Number CE140100012) is gratefully acknowledged.

\section{References}

1. Hammer N, Glätzner J, Feja C, et al. Human Vagus Nerve Branching in the Cervical Region. PLoS One. e0118006 (2015).

2. Thompson N, Mastitskaya S, Holder D. Avoiding off-target effects in electrical stimulation of the cervical vagus nerve: Neuroanatomical tracing techniques to study fascicular anatomy of the vagus nerve. J. Neurosci. Methods [Internet]. 325, 108325 (2019). Available from: http://www.sciencedirect.com/science/article/pii/S0165027019301839.

3. Volta A. XVII. On the electricity excited by the mere contact of conducting substances of different kinds. In a letter from Mr. Alexander Volta, F. R. S. Professor of Natural Philosophy in the University of Pavia, to the Rt. Hon. Sir Joseph Banks, Bart. K.B. P. R. Philos. Trans. R. Soc. London [Internet]. 90, 403-431 (1800). Available from: http://rstl.royalsocietypublishing.org/content/90/403.short.

4. Sarnoff SJ, Gaensler EA, Maloney Jr J V. Electrophrenic respiration; IV. The effectiveness of contralateral ventilation during activity of one phrenic nerve. J. Thorac. Surg. 19(6), 929 (1950).

5. Djourno A, Eyriès C. Prothese auditive par excitation electrique a distance du nerf sensoriel a laide dun bobinage inclus a demeure. Press. médicale. 65(63), 1417 (1957).

6. Eisen M. Djourno, Eyries, and the First Implanted Electrical Neural Stimulator to Restore Hearing. Otol Neurotol [Internet]. 24(3), 500-506 (2003). Available from: http://ovidsp.ovid.com/ovidweb.cgi?T=JS\&PAGE=reference $\& D=0 v f t \& N E W S=N \& A N=001$ 29492-200305000-00025.

7. House WF, Urban J. Long term results of electrode implantation and electronic stimulation of the cochlea in man. Ann. Otol. Rhinol. Laryngol. 82, 504-517 (1973).

8. Clark GM, Black R, Dewhurst DJ, Forster IC, Patrick JF, Tong YC. A multiple-electrode hearing prosthesis for cochlea implantation in deaf patients. Med. Prog. Technol. [Internet]. 
5(3), 127-140 (1977). Available from: http://hdl.handle.net/11343/27158.

9. Liberson WT, Holmquest HJ, Scot D, Dow M. Functional electrotherapy: stimulation of the peroneal nerve synchronized with the swing phase of the gait of hemiplegic patients. Arch. Phys. Med. Rehabil. [Internet]. 42, 101-5 (1961). Available from: http://www.ncbi.nlm.nih.gov/pubmed/13761879.

10. Shealy CN, Mortimer JT, Reswick JB. Electrical Inhibition of Pain by Stimulation of the Dorsal Columns: Preliminary Clinical Report. Anesth. Analg. [Internet]. 46(4) (1967). Available from: https://journals.lww.com/anesthesiaanalgesia/Fulltext/1967/07000/Electrical_Inhibition_of_Pain_by_Stimulation_of.25.aspx.

11. LeRoy C. Ou l'on rend compte de quelques tentatives que l'on a faites pour guerir plusieurs maladies par l'electricite. Mem. Math Phys [Internet]. 60, 87-95 (1755). Available from: $\mathrm{http} / / /$ scholar.google.com/scholar?as_q=\&as_epq=Où l'on rend compte de quelques tentatives que l'on a faites pour guérir plusieurs maladies par

l'électricité\&as_oq $=\&$ as_eq $=\&$ as_occt $=$ any\&as_sauthors $=$ LeRoy $\& a s \_p u b l i c a t i o n=\& a s \_y l o=$ \&as_yhi $=\& b \operatorname{tnG}=\& h l=e n \&$ sciui $=1 \&$ as.

12. Chuang AT, Margo CE, Greenberg PB. Retinal implants: a systematic review. Br. J. Ophthalmol. [Internet]. 98(7), 852 LP - 856 (2014). Available from: http://bjo.bmj.com/content/98/7/852.abstract.

13. Corning J. Electrization of the Sympathetic and pneumogastric nerves, with simultaneous bilateral compression of the carotids. New York Med. J. 39, 212-215 (1884).

14. Yuan H, Silberstein SD. Vagus Nerve and Vagus Nerve Stimulation, a Comprehensive Review: Part III. Headache J. Head Face Pain [Internet]. 56(3), 479-490 (2016). Available from: https://doi.org/10.1111/head.12649.

15. Horn CC, Ardell JL, Fisher LE. Electroceutical Targeting of the Autonomic Nervous System. Physiology [Internet]. 34(2), 150-162 (2019). Available from: https://doi.org/10.1152/physiol.00030.2018.

16. Naufel S, Knaack GL, Miranda R, et al. DARPA investment in peripheral nerve interfaces for prosthetics, prescriptions, and plasticity. J. Neurosci. Methods [Internet]. 332, 108539 (2020). Available from: http://www.sciencedirect.com/science/article/pii/S0165027019303966.

17. Drennan WP, Rubinstein JMDP. Music perception in cochlear implant users and its relationship with psychophysical capabilities. J. Rehabil. Res. Dev. [Internet]. 45(5), 779 (2008). Available from:

http://proquest.umi.com/pqdweb?did=1566219401\&Fmt=7\&clientId=20828\&RQT=309\&VN ame=PQD.

18. Ohemeng KK, Parham K. Vagal Nerve Stimulation: Indications, Implantation, and Outcomes. Otolaryngol. Clin. North Am. [Internet]. 53(1), 127-143 (2020). Available from: http://www.sciencedirect.com/science/article/pii/S0030666519301756.

19. Horch KW, Dhillon GS. Neuroprosthetics: Theory and Practice [Internet]. World Scientific. Available from: https://books.google.com.au/books?id=jrpeu7eASn0C.

20. Franz S, Rammelt S, Scharnweber D, Simon JC. Immune responses to implants - A review of the implications for the design of immunomodulatory biomaterials. Biomaterials [Internet]. 32(28), 6692-6709 (2011). Available from:

http://www.sciencedirect.com/science/article/pii/S0142961211006491.

21. Williams DF. On the mechanisms of biocompatibility. Biomaterials [Internet]. 29(20), 29412953 (2008). Available from:

http://www.sciencedirect.com/science/article/pii/S0142961208002676.

22. Seligman P. Prototype to product - developing a commercially viable neural prosthesis. $J$. Neural Eng. [Internet]. 6(6), 65006 (2009). Available from: http://stacks.iop.org/1741$2552 / 6 / \mathrm{i}=6 / \mathrm{a}=065006$.

23. Dhanasingh A, Jolly C. An overview of cochlear implant electrode array designs. Hear. Res. [Internet]. 356(Supplement C), 93-103 (2017). Available from: http://www.sciencedirect.com/science/article/pii/S0378595517302940.

24. Stronks HC, Dagnelie G. The functional performance of the Argus II retinal prosthesis. Expert Rev. Med. Devices [Internet]. 11(1), 23-30 (2014). Available from:

https://www.ncbi.nlm.nih.gov/pubmed/24308734. 
25. Weiland JD, Humayun MS. Retinal prosthesis. IEEE Trans. Biomed. Eng. [Internet]. 61(5), 1412-1424 (2014). Available from: https://www.ncbi.nlm.nih.gov/pubmed/24710817.

26. Stingl K, Bartz-Schmidt KU, Besch D, et al. Subretinal Visual Implant Alpha IMS - Clinical trial interim report. Vision Res. [Internet]. 111, 149-160 (2015). Available from:

http://www.sciencedirect.com/science/article/pii/S0042698915000784.

27. Bradley K. The Technology: The Anatomy of a Spinal Cord and Nerve Root Stimulator: The Lead and the Power Source. Pain Med. [Internet]. 7(suppl_1), S27-S34 (2006). Available from: https://dx.doi.org/10.1111/j.1526-4637.2006.00120.x.

28. Larson CE, Meng E. A review for the peripheral nerve interface designer. J. Neurosci. Methods [Internet]. 332, 108523 (2020). Available from: http://www.sciencedirect.com/science/article/pii/S0165027019303802.

29. Badia J, Boretius T, Andreu D, Azevedo-Coste C, Stieglitz T, Navarro X. Comparative analysis of transverse intrafascicular multichannel, longitudinal intrafascicular and multipolar cuff electrodes for the selective stimulation of nerve fascicles. J. Neural Eng. [Internet]. 8(3), 36023 (2011). Available from: http://stacks.iop.org/1741-2552/8/i=3/a=036023.

30. Tykocinski M, Duan Y, Tabor B, Cowan RS. Chronic electrical stimulation of the auditory nerve using high surface area (HiQ) platinum electrodes. Hear. Res. [Internet]. 159(1-2), 5368 (2001). Available from:

http://www.sciencedirect.com/science/article/pii/S0378595501003203.

31. Schiavone G, Wagner F, Fallegger F, et al. Long-term functionality of a soft electrode array for epidural spinal cord stimulation in a minipig model. In: 2018 40th Annual International Conference of the IEEE Engineering in Medicine and Biology Society (EMBC), 1432-1435 (2018).

32. Yu X, Su JY, Guo JY, et al. Spatiotemporal characteristics of neural activity in tibial nerves with carbon nanotube yarn electrodes. J. Neurosci. Methods [Internet]. 328, 108450 (2019). Available from: http://www.sciencedirect.com/science/article/pii/S0165027019303073.

33. Wang K, Frewin CL, Esrafilzadeh D, et al. High-Performance Graphene-Fiber-Based Neural Recording Microelectrodes. Adv. Mater. [Internet]. 0(0), 1805867 (2019). Available from: https://doi.org/10.1002/adma.201805867.

34. Gorman PH, Mortimer JT. The Effect of Stimulus Parameters on the Recruitment Characteristics of Direct Nerve Stimulation. IEEE Trans. Biomed. Eng. BME-30(7), 407-414 (1983).

35. Harris AR, Wallace GG. Organic Electrodes and Communications with Excitable Cells. Adv. Funct. Mater. [Internet]. 28(12), 1700587 (2018). Available from: http://dx.doi.org/10.1002/adfm.201700587.

36. Agnew WF, McCreery DB. Considerations for Safety with Chronically Implanted Nerve Electrodes. Epilepsia [Internet]. 31(s2), S27-S32 (1990). Available from: https://doi.org/10.1111/j.1528-1157.1990.tb05845.x.

37. Shannon R V. A model of safe levels for electrical stimulation. Biomed. Eng. IEEE Trans. 39(4), $\operatorname{cog}(1992)$.

38. McCreery D, Agnew W, Yuen T, Bullara L. Comparison of neural damage induced by electrical stimulation with faradaic and capacitor electrodes. Ann. Biomed. Eng. [Internet]. 16(5), 463-481 (1988). Available from: http://dx.doi.org/10.1007/BF02368010.

39. McCreery DB, Agnew WF, Yuen TGH, Bullara L. Charge density and charge per phase as cofactors in neural injury induced by electrical stimulation. Biomed. Eng. IEEE Trans. [Internet]. 37(10), 996-1001 (1990). Available from: http://10.0.4.85/10.102812.

40. Cogan SF, Ludwig KA, Welle CG, Takmakov P. Tissue damage thresholds during therapeutic electrical stimulation. J. Neural Eng. [Internet]. 13(2), 21001 (2016). Available from: http://stacks.iop.org/1741-2552/13/i=2/a=021001.

41. McCreery D, Agnew W, Yuen T, Bullara L. Damage in peripheral nerve from continuous electrical stimulation: Comparison of two stimulus waveforms. Med. Biol. Eng. Comput. [Internet]. 30(1), 109-114 (1992). Available from: http://dx.doi.org/10.1007/BF02446202.

42. McCreery DB, Agnew WF, Yuen TGH, Bullara LA. Relationship between stimulus amplitude, stimulus frequency and neural damage during electrical stimulation of sciatic nerve of cat. Med. Biol. Eng. Comput. [Internet]. 33(3), 426-429 (1995). Available from: 
https://doi.org/10.1007/BF02510526.

43. Walsh SM, Leake-Jones PA. Chronic electrical stimulation of auditory nerve in cat: Physiological and histological results. Hear. Res. [Internet]. 7(3), 281-304 (1982). Available from: http://www.sciencedirect.com/science/article/pii/0378595582900417.

44. Shepherd RK, Matsushima J, Martin RL, Clark GM. Cochlear pathology following chronic electrical stimulation of the auditory nerve: II deafened kittens. Hear. Res. [Internet]. 81(1-2), 150-166 (1994). Available from:

http://www.sciencedirect.com/science/article/pii/0378595594901627.

45. Clark GM, Clark J, Cardamone T, et al. Biomedical studies on temporal bones of the first multi-channel cochlear implant patient at the University of Melbourne. abid [Internet]. 15(sup2), S1-S15 (2014). Available from: https://doi.org/10.1179/1754762814Y.0000000087.

46. Shepherd RK, Carter PM, Enke YL, Wise AK, Fallon JB. Chronic intracochlear electrical stimulation at high charge densities results in platinum dissolution but not neural loss or functional changes in vivo. J. Neural Eng. [Internet]. 16(2), 26009 (2019). Available from: http://dx.doi.org/10.1088/1741-2552/aaf66b.

47. Günter C, Delbeke J, Ortiz-Catalan M. Safety of long-term electrical peripheral nerve stimulation: Review of the state of the art. J. Neuroeng. Rehabil.16(1) (2019).

48. Gunasekera B, Saxena T, Bellamkonda R, Karumbaiah L. Intracortical Recording Interfaces: Current Challenges to Chronic Recording Function. ACS Chem. Neurosci. [Internet]. 6(1), 6883 (2015). Available from: http://dx.doi.org/10.1021/cn5002864.

49. Wang Y, Qin Z. Molecular and cellular mechanisms of excitotoxic neuronal death. Apoptosis [Internet]. 15(11), 1382-1402 (2010). Available from: https://doi.org/10.1007/s10495-0100481-0.

50. Harris AR, Wallace GG. Electrochemical Methods for Analysing and Controlling Charge Transfer at the Electrode-Tissue Interface. Curr. Opin. Electrochem. 16, 143-148 (2019).

51. Harris AR, Newbold C, Carter P, Cowan R, Wallace GG. Measuring the Effective Area and Charge Density of Platinum Electrodes for Bionic Devices. J. Neural Eng. [Internet]. 15(4), 46015 (2018). Available from: https://www.ncbi.nlm.nih.gov/pubmed/29595147.

52. Harris AR, Newbold C, Carter P, Cowan R, Wallace GG. Using Chronopotentiometry to Better Characterize the Charge Injection Mechanisms of Platinum Electrodes Used in Bionic Devices. Front. Neurosci. [Internet]. 13, 380 (2019). Available from: https://www.frontiersin.org/article/10.3389/fnins.2019.00380/full.

53. Wissel K, Brandes G, Pütz N, et al. Platinum corrosion products from electrode contacts of human cochlear implants induce cell death in cell culture models. PLoS One [Internet]. 13(5), e0196649 (2018). Available from: https://doi.org/10.1371/journal.pone.0196649.

54. Kovach KM, Kumsa DW, Srivastava V, et al. High-throughput in vitro assay to evaluate the cytotoxicity of liberated platinum compounds for stimulating neural electrodes. J. Neurosci. Methods [Internet]. 273, 1-9 (2016). Available from: http://www.sciencedirect.com/science/article/pii/S0165027016301790.

55. Kumsa DW, Hudak EM, Bhadra N, Mortimer JT. Electron transfer processes occurring on platinum neural stimulating electrodes: pulsing experiments for cathodic-first, chargeimbalanced, biphasic pulses for $0.566 \leqslant \mathrm{k} \leqslant 2.3$ in rat subcutaneous tissues. J. Neural Eng. [Internet]. 16(2), 26018 (2019). Available from: http://dx.doi.org/10.1088/1741-2552/aaf931.

56. Robblee LS, McHardy J, Marston JM, Brummer SB. Electrical stimulation with Pt electrodes. V. The effect of protein on Pt dissolution. Biomaterials [Internet]. 1(3), 135-139 (1980). Available from: http://www.sciencedirect.com/science/article/B6TWB-48H34W12T/2/34f5042dc585c6cd669cbc70ce2f757b.

57. Brummer SB, McHardy J, Turner MJ. Electrical stimulation with Pt electrodes: trace analysis for dissolved platinum and other dissolved electrochemical products. Brain. Behav. Evol. 14, 10-22 (1977).

58. Hibbert DB, Weitzner K, Tabor B, Carter P. Mass changes and dissolution of platinum during electrical stimulation in artificial perilymph solution. Biomaterials [Internet]. 21(21), 21772182 (2000). Available from: http://www.sciencedirect.com/science/article/pii/S0142961200001460.

59. Mitsushima S, Kawahara S, Ota K, Kamiya N. Consumption Rate of Pt under Potential 
Cycling. J. Electrochem. Soc. [Internet]. 154(2), B153-B158 (2007). Available from: http://jes.ecsdl.org/content/154/2/B153.abstract.

60. Harris AR, Newbold C, Cowan R, Wallace GG. Insights into the Electron Transfer Kinetics, Capacitance and Resistance Effects of Implantable Electrodes Using Fourier Transform AC Voltammetry on Platinum. J. Electrochem. Soc. [Internet]. 166(12), G131-G140 (2019). Available from: http://jes.ecsdl.org/content/166/12/G131.abstract.

61. Merrill DR, Bikson M, Jefferys JGR. Electrical stimulation of excitable tissue: design of efficacious and safe protocols. J. Neurosci. Methods [Internet]. 141(2), 171-198 (2005). Available from: http://www.sciencedirect.com/science/article/B6T04-4F53NB61/2/b3f4360309a1bc8da76c639ee69e9dc3. 


\section{University Library}

\section{- M M I N E R VA A gateway to Melbourne's research publications}

Minerva Access is the Institutional Repository of The University of Melbourne

Author/s:

Harris, AR

Title:

Current perspectives on the safe electrical stimulation of peripheral nerves with platinum electrodes

Date:

2020-09

\section{Citation:}

Harris, A. R. (2020). Current perspectives on the safe electrical stimulation of peripheral nerves with platinum electrodes. Bioelectronics in Medicine, 3 (3), pp.37-49. https:// doi.org/10.2217/bem-2020-0007.

Persistent Link:

http://hdl.handle.net/11343/274299 